\title{
THE CYLINDRICAL ANTENNA WITH GAP
}

\author{
BY \\ RONOLD KING AND T. W. WINTERNITZ \\ Cruft Laboratory and The Lyman Laboratory of Physics, Harvard University
}

1. Introduction. In the King-Middleton theory of the center-driven cylindrical antenna ${ }^{1}$ current and impedance are defined in terms of a discontinuity in scalar potential. Such a driving mechanism is unavailable physically, since actual potential differences necessarily exist across finite distances. In practice, an antenna usually is connected to the conductors of a transmission line across which a potential difference is maintained by a generator elsewhere along the line. A common arrangement is shown in Fig. 1. The antenna in Fig. 1 differs from a conductor center-driven by a discontinuity in scalar potential in several respects. First, there is a gap in place of a

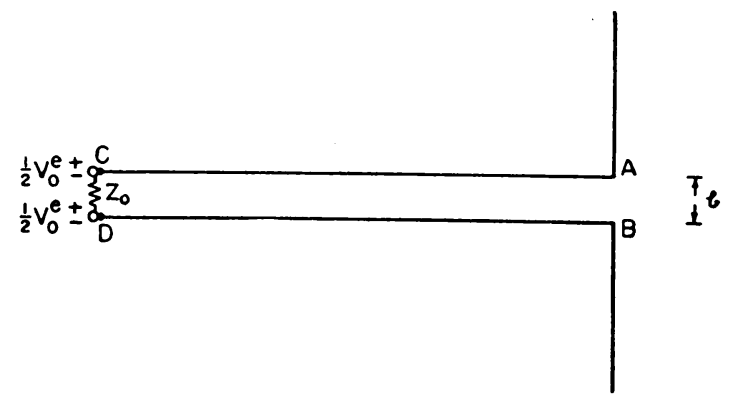

Fig. 1. Antenna center-driven from a two-wire line.

current-carrying section of conductor in the region between $A$ and $B$ or $z=-\delta$ and $z=\delta$. Second, a part of the space around the antenna is occupied by the two conductors of the transmission line carrying equal and opposite distributions of current and charge separated a finite distance $b \doteq 2 \delta$. Third, there exist small junction regions (of magnitude comparable with the radius $a$ of the conductors) where each half of the antenna joins one of the conductors of the line. These regions are not obviously a part of either the antenna alone or the line alone, and thus introduce an element of vagueness as to where the antenna ends and the line begins.

The rigorous analysis of the complete circuit of Fig. 1 (as of most other electric networks including even a simple series circuit consisting of a coil and a condenser) is excessively difficult if formulated as a three-dimensional boundary-value problem in electromagnetic field-theory. Fortunately, it is possible to gain much in simplicity at the price of a measure of rigor by requiring the radius $a$ of all conductors to be sufficiently small to satisfy the inequality,

$$
\beta_{0}=\omega a / v_{0}=2 \pi a / \lambda_{0} \ll 1 .
$$

Subject to (1), it is a good approximation to assume that cross-sectional and axial distributions of current in conductors are independent, and so reduce the analysis of complex configurations to one-dimensional form.

${ }^{1}$ R. King and D. Middleton, Quart. Appl. Math. 3, 302 (1946). 
2. One dimensional theory. The general formulation of one-dimensional electric circuit and antenna theory is available in the literature.$^{1,2,3,4,5} \mathrm{It}$ is based on the fundamental relations,

$$
\begin{gathered}
-\operatorname{grad} \varphi-j \omega \mathbf{A}=\mathbf{E} \\
\operatorname{div} \mathbf{A}+j \frac{\beta_{0}^{2}}{\omega} \varphi=0
\end{gathered}
$$

where $\mathbf{A}$ is the Lorentz vector potential defined in general by (3) in conjunction with

$$
\operatorname{curl} \mathbf{A}=B \text {. }
$$

A particular integral of the non-homogeneous wave equation in $\mathbf{A}$ obtained by substituting (2), (3), (4) into the Maxwell equation is

$$
\mathrm{A}=\frac{1}{4 \pi \nu_{0}}\left\{\int_{\tau} \frac{\mathrm{i}^{\prime}}{R} e^{-j \beta_{0} R} d \tau^{\prime}+\int_{\Sigma} \frac{\mathbf{1}^{\prime}}{R} e^{-j \beta_{0} R} d \sigma^{\prime}\right\} .
$$

where $\nu_{0}=1 / \mu_{0}=10^{7} / 4 \pi$ meters/henry, $i$ is the volume density of current, and 1 the surface density of current. $R$ is the distance from the unprimed coordinate of the point where $\mathbf{A}$ is calculated to the primed coordinates locating the elements of integration. In good conductors, only $i$ is used; in perfect conductors, $i$ is zero and 1 is required. Integration is carried out over all conductors of the circuit so that the vector potential $\mathbf{A}$ is uniquely defined by (5) and the scalar potential $\varphi$ by (3).

For simplicity, let it be assumed that the dielectric is air and that the conductors may be treated as perfect in writing the following boundary conditions for the tangential component of the electric field:

$$
\begin{aligned}
& \frac{\partial \varphi}{\partial z}+j \omega A_{z}=-E_{z} \doteq 0 \text { on the surface of the antenna; } \\
& \frac{\partial \varphi}{\partial x}+j \omega A_{x}=-E_{x} \doteq 0 \text { on the surface of each line conductor. }
\end{aligned}
$$

Since there are no currents in the $y$-direction, $A_{y}$ vanishes everywhere. Hence, (3) gives

$$
\frac{\partial A_{z}}{\partial z}+\frac{\partial A_{x}}{\partial x}+j \frac{\beta_{0}^{2}}{\omega} \varphi=0
$$

on the antenna and on the line. Differentiation of (8) with respect to $z$ and substitution from (6) gives the following equation:

$$
\frac{\partial^{2} A_{z}}{\partial z^{2}}+\frac{\partial^{2} A_{x}}{\partial z \partial x}+\beta_{0}^{2} A_{x}=0
$$

2 R. W. P. King, Electromagnetic engineering, McGraw-Hill Book Co., New York, 1945, Vol. 1, Ch. VI and pp. 193-195.

${ }^{3}$ J. R. Carson, Bell Syst. Tech. Journal 6, No. 1 (1927).

4 A. T. Starr, Electric circuits and wave filters, Pitman Publishing Corp., New York, 1938, Ch. II, especially pp. 41-53.

${ }^{5} \mathrm{~J}$. Aharoni, Antennae, Oxford Univ. Press, 1946, Ch. II. 
on the antenna. Similarly, differentiation of (8) with respect to $x$ and substitution from (7) gives

$$
\frac{\partial^{2} A_{x}}{\partial x^{2}}+\frac{\partial^{2} A_{z}}{\partial x \partial z}+\beta_{0}^{2} A_{x}=0
$$

on each conductor of the line.

Since it has been assumed in (1) that all conductors have a radius $a$ that is sufficiently small so that $\beta_{0} a \ll 1$ is a good approximation, the contributions to the vector potential at all outside points (including the surface of the conductor) due to the radial components of current in that conductor may be neglected compared with the contributions due to the axial components of current. This is equivalent to assuming that the currents in the antenna set up only a $z$-component of the vector potential, the currents in the transmission line only an $x$-component of $\mathbf{A}$. It has been shown ${ }^{6,7}$ that if the current in a cylindrical conductor has rotational symmetry and the conductor is long compared with its radius, the vector potential on the surface of the conductor differs negligibly from the value it would have if the total current in the conductor were concentrated on the axis instead of being distributed in the manner predicted by skin-effect theory. That is, the component of the Lorentz potential on the surface of the antenna due to the axial currents in the antenna is given by

$$
A_{z}=\frac{1}{4 \pi \nu_{0}}\left\{\int_{-h}^{-\delta} \frac{I_{z}^{\prime}}{R_{a}} e^{-j \beta_{0} R_{a}} d z^{\prime}+\int_{\delta}^{h} \frac{I_{z}^{\prime}}{R_{a}} e^{-j \beta_{0} R_{a}} d z^{\prime}\right\}
$$

Similarly, on the surface of one of the conductors of the line, the component of the Lorentz potential due to the equal and opposite currents in both conductors is given by:

$$
A_{x}=\frac{1}{4 \pi \nu_{0}} \int_{0}^{s} I_{x}^{\prime}\left[\frac{e^{-j \beta_{0} R_{a}}}{R_{a}}-\frac{e^{-i \beta_{0} R_{b}}}{R_{b}}\right] d x^{\prime} .
$$

Since $A_{x}$ is determined entirely from currents in the line and $A_{z}$ entirely from currents in the antenna, the terms with mixed derivatives in (9) and (10) represent coupling between line and antenna. This is significant within distances from $A$ and $B$ (Fig. 1) that are not great compared with $b$. At distances from $A$ and $B$ which are great compared with $b$, these terms are both negligible and the two equations reduce to

$$
\begin{aligned}
& \frac{\partial^{2} A_{z}}{\partial z^{2}}+\beta_{0}^{2} A_{z}=0 \text { on the surface of the antenna, } \\
& \frac{\partial^{2} A_{x}}{\partial x^{2}}+\beta_{0}^{2} A_{x}=0 \text { on the surface of each conductor of the line. }
\end{aligned}
$$

' Ref. 2, pp. 239-243.

${ }^{7}$ O. Zinke, Archiv für Elektrotechnik, 35, 67 (1941). This work of Zinke shows that the contributions to $A_{z}$ at $z$ due to currents in different elements $d z^{\prime}$ at $z^{\prime}$ depend upon an effective radius $r_{w}$ that is smaller than $a$ for $\left|z-z^{\prime}\right|<0.4 a$ and larger than $a$ for $\left|z-z^{\prime}\right|>0.4 a$. The value of $\int_{-s}^{\prime} d z^{\prime} / R_{w}$ where

$$
R_{w}=\sqrt{\left(z-z^{\prime}\right)^{2}+r_{w}^{2}}
$$

differs from $\int_{s}^{a} d z^{\prime} / R_{a}$, where $R_{a}=\sqrt{\left(z-z^{\prime}\right)^{2}+a^{2}}$ by less than $1 \%$ if $s \geqq 2.5 a$ for high frequency values of skin depth in copper. 
Of these equations, the first is the one solved in the King-Middleton analysis of the cylindrical antenna ${ }^{1}$ by an extension of the method of Hallen $;^{8}$ the second is that used in the fundamental derivation of the conventional transmission-line equations for current and voltage. ${ }^{9}$

The derivation of the conventional transmission-line equation for current from (14) involves certain approximations since the current does not actually satisfy the transmission-line equation exactly, even for perfect conductors, as do both the vector and scalar potentials. The current can be made to satisfy the transmission-line equations approximately only if the effect of radiation on the distribution of current is made negligible by imposing the inequality,

$$
\beta_{0} b \ll 1,
$$

and if, in addition, it is a good approximation to set

$$
I_{x}=\left[\left(A_{x}\right)_{1}+\left(A_{x}\right)_{2}\right] / l_{0}^{e}(x)
$$

where

$$
l_{0}^{e}(x)=\frac{1}{\pi \nu_{0}}\left[\ln \frac{b}{a}-\frac{1}{2} \ln F(x)\right] \doteq \frac{1}{\pi \nu_{0}} \ln \frac{b}{a}=l_{0}^{e} .
$$

The function $F(x)$ reduces practically to unity ${ }^{10}$ at distances from the ends of the line which are great compared with $b$. Only when this is true, i.e., when

$$
(s-x)^{2} \gg b^{2} ; \quad x^{2} \gg b^{2} ;
$$

does (17) give the conventional external inductance per unit lergth of line, $l_{0}^{e}$. On the other hand, at the ends where $x=0$ or $s, F(x)=b / a$, so that $l_{0}^{e}(x)$ is only one half of $l_{0}^{e}$, its normal value. ${ }^{11}$ Correspondingly, the capacitance per unit length, $c=\epsilon_{0} \mu_{0} / l_{0}^{e}(x)$, becomes double the value far from the ends. Only when both (15) and (18) are satisfied does the current obey the transmission-line equations to a good approximation.

Summarizing, it is clear that subject to $\beta_{0} a<\beta_{0} b \ll 1$ the conventional transmission-line equations in the total current and the potential difference are good approximations only at distances from the ends of the line that are reasonably large compared with the distance $b$ between conductors. At such distances the line parameters are quite constant and, in addition, the coupling between line and antenna due to the mixed derivative terms in (9) and (10) is negligible.

From the point of view of a three-dimensional boundary value problem in field theory in which the boundary conditions of the terminated line are satisfied using an expansion in terms of characteristic wave functions, the conditions (18) that make the conventional line equations good approximations are equivalent to specifying distances from the ends of the line that are sufficiently great to make all exponentially damped higher modes negligible. Current and voltage are then represented to a good approximation by the dominant transmission-line mode. ${ }^{12}$

${ }^{8} \mathrm{E}$. Hallén, Theoretical investigations into the transmitting and receiving qualities of antennae, Nova Acta Uppsala, (4) 11, No. 4 (1938).

- See for example, Ref. 2, pp. 468-477.

${ }^{10}$ Ref. 2, p. 473 , Eq. 185.

${ }^{11}$ E. M. Siegel, Univ. of Texas Publ., No. 4031 (1940).

12 J. R. Carson, A.I.E.E. Journal, 43, 908 (1924). 
3. Definition of impedance. Since the antenna in the circuit of Fig. 1 has been shown to be coupled to the adjacent parts of the transmission line, it is not possible to define for the antenna an impedance $Z_{A B}=Z_{\delta}$ that is a function only of its own physical properties. It is, of course, possible to define the impedance of the antenna and an attached short section of transmission line that is sufficiently long so that coupling to the termination and end effects are negligible. The impedance so defined
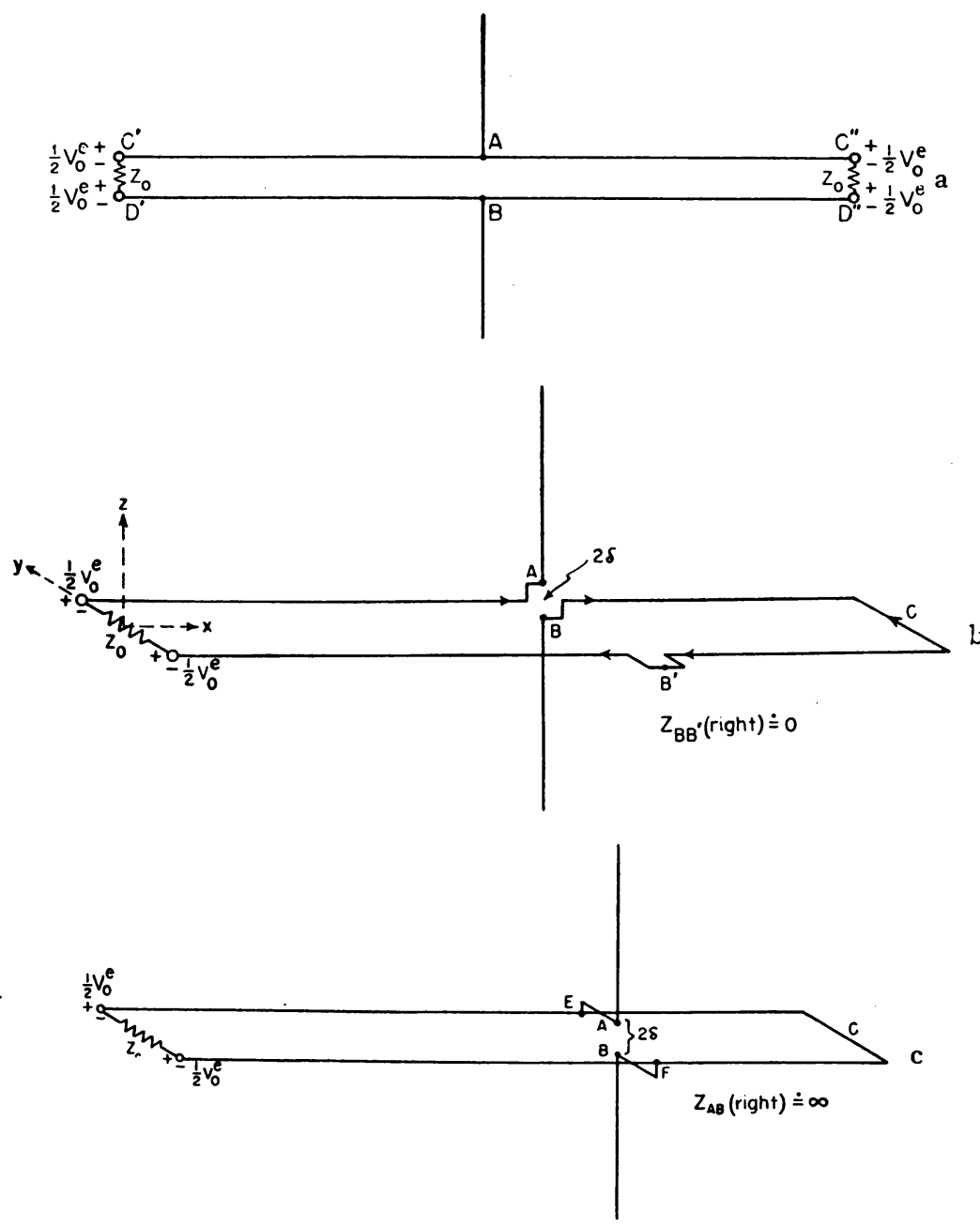

FIGs. 2a, b, c. Antenna center-driven in different ways from a two-wire line.

may then be transformed to the actual location of the antenna using conventional line theory. This is the procedure commonly used both for wave guides and conventional lines in determining equivalent impedances or equivalent lumped-constant networks for discontinuities or terminations. Evidently an impedance so determined is not a characteristic property of the termination alone, e.g. the antenna in Fig. 1, but is that fictitious impedance that would have to terminate a particular line if this had the physically impossible property of having the conventional line equations everywhere 
rigorously true, including regions near the junction with the termination. The investigation of such equivalent or apparent impedances for an antenna driven in various ways from two-wire and coaxial lines is in progress both theoretically and experimentally. Three arrangements for a two-wire line different from that in Fig. 1 are shown in Figs. 2a, b, c. A moment's reflection suggests that the mixed derivative terms in (9) and (10), as well as the ratio $\left[\left(A_{x}\right)_{1}+\left(A_{x}\right)_{2}\right] / I_{x}$ may have quite different values in the four circuits in Figs. 1 and 2 near the antenna. Therefore, it is not to be expected that the equivalent dominant mode impedances will be the same in the four cases even when the antennas themselves and the gap between their halves are made identical. The quantitative effect of these special differences is not the subject of this paper.

The present purpose is to generalize the King-Middleton theory as much as possible without limiting it specifically to one of the circuits shown in Figs. 1 and 2, or to any other. What can be determined in general is the effect on the current and impedance of a cylindrical antenna with hemispherical caps of a gap of length $2 \delta$ between the halves of the antenna, across which a scalar potential difference $V_{\delta}$ is maintained in an unspecified manner. The driving potential difference is to be measured between adjacent rings on the cylindrical surfaces of the two halves of the antenna. Depending upon the nature of the actual driving circuit, there may be exposed flat-end surfaces if the cylinders are solid, or inner surfaces and sharp edges if they are tubes, or elbows, or other junction regions. These are not included in the analysis. In simple cases, they may constitute a capacitance in parallel with the antenna, or they may be part of a four-terminal network, or account may be taken of them in other ways in the analysis of an actual circuit such as is shown in Figs. 1 and 2. In any event, they constitute a part of the problem of a particular complete circuit, not of the general effect of a separation of the halves of a symmetrical antenna insofar as the interaction of currents and charges in its cylindrical surfaces are concerned. These alone are considered here.

The impedance calculated in the next section is, by definition, the ratio of the scalar potential difference maintained across equipotential rings separated a distanco $2 \delta$ and the total axial current entering the antenna across planes bounded by these rings. In good conductors, most of the current is in a relatively thin layer near the surface so that, as shown by Zinke, ${ }^{7}$ the entire current may be assumed concentrated at one half the skin depth. (Note that the vector potential on the surface of the conductor is always defined outside the region of non-vanishing current density.) The impedance is defined as follows: $:^{13}$

${ }^{13}$ A definition of impedance in terms of scalar potential difference instead of the line integral of the electric field as used by other writers ${ }^{14,15,16,17}$ is preferred because the scalar potential difference is uniquely defined, whereas the line integral of the electric field depends upon the path of integration. The line integral of the electric field coincides with the scalar potential difference only when a path is chosen along which $\omega$ times the line integral of the vector potential vanishes or is negligible, as along a radial path in transmission lines sufficiently far from the termination. It is difficult to see how in conventional one-dimensional circuits involving conductors of small cross-section the impedance of a part of a circuit connected to terminals that are separated a finite distance can be defined consistently except in terms of the scalar potential difference maintained across those terminals. For example, the reactive drop across a loosely wound coil of a few turns of wire is not correctly given by the line integral of the electric field along any path. This is most easily seen if the coil degenerates into a straight conductor when the line integral of the electric field along the conductor does not include the inductive reactive drop at all, although it is the 


$$
Z_{\delta}=\frac{V_{\delta}}{I_{\delta}}, \quad V_{\delta}=\varphi(\delta)-\varphi(-\delta) .
$$

4. Theoretical analysis. The integral equation for the current in the cylindrical antenna which has its halves separated by a gap $2 \delta$ differs from the former Eq. (8), Ref. 1, primarily in the limits of integration and in the phase of the cosine function. Thus, the equation for the upper half of the antenna is:

$$
\begin{aligned}
4 \pi \nu_{0} A_{z} & =\int_{-h}^{-\delta} I_{z^{\prime}} R^{-1} e^{-j \beta_{0} R} d z^{\prime}+\int_{\delta}^{h} I_{z^{\prime}} R^{-1} e^{-i \beta_{0} R} d z^{\prime} \\
& =\frac{j 4 \pi}{R_{c} \cos \beta_{0} \delta}\left[C_{1} \cos \beta_{0}(z-\delta)+\frac{1}{2} V_{\delta} \sin \beta_{0} z-z^{i} \cos \beta_{0} \delta \int_{\delta}^{z} I(s) \sin \beta_{0}(z-s) d s\right] \\
& (\delta \leqq z \leqq h) .
\end{aligned}
$$

The following symmetry conditions are satisfied:

$$
I(-z)=I(z) ; \quad A_{z}(-z)=A_{z}(z) .
$$

For perfect conductors $z^{i}=0$. The solution of (20) is carried out by the same method of successive approximations. The $m$ th order current corresponding to (24) of Ref. 1, and to which it reduces with $\delta=0$, is

$$
\begin{gathered}
\left(I_{z}\right)_{m}=\frac{j 2 \pi V_{\delta}}{R_{c} \psi} \cdot \frac{\sin \beta_{0}(h-z)+\sum_{n=1}^{m} M_{n}^{\delta}(z) / \psi^{n}}{\cos \beta_{0}(h-\delta)+\cos \beta_{0} \delta \sum_{n=1}^{m} F_{n}^{\delta}(h) / \psi^{n}+\sin \beta_{0} \delta \sum_{n=1}^{m} G_{n}^{\delta}(h) / \psi^{n}} \\
(\delta \leqq z \leqq h) .
\end{gathered}
$$

The functions $M_{n}^{\delta}(z), F_{n}^{\delta}(h)$, and $G_{n}^{\delta}(h)$ are like the functions $M_{n}(z), F_{n}(h)$, and $G_{r}(h)$ previously defined but with limits of integration from $-h$ to $-\delta, \delta$ to $h$, instead of from $-h$ to $h$.

The $m$ th order impedance corresponding to (29) in Ref. 1 is

$$
\left(Z_{\delta}\right)_{m}=\frac{j R_{c} \psi}{2 \pi} \frac{\cos \beta_{0}(h-\delta)+\cos \beta_{0} \delta \sum_{n=1}^{m} F_{n}^{\delta}(h) / \psi^{n}+\sin \beta_{0} \delta \sum_{n=1}^{m} G_{n}^{\delta}(h) / \psi^{n}}{\sin \beta_{0}(h-\delta)+\sum_{n=1}^{m} M_{n}^{\delta}(\delta) / \psi^{n}} .
$$

principle contribution to the impedance if the conductor is copper. On the other hand, if the scalar potential difference is used both ohmic resistance and inductive reactance drops are included.

In this connection, it is well to note that the field impedances in wave-guide theory defined in terms of line integrals of the electric field are not simply generalizations of circuit impedances in one-dimensional theory. Rather, they are quantities that have properties analogous to those of circuit impedances but in terms of quite different and much more arbitrary variables than are scalar potential difference and total current in conductors of small cross-section.

14 J. A. Stratton and L. J. Chu, J. Appl. Phys. 12, 230 (1941).

${ }^{15}$ S. A. Schelkunoff, Proc. I.R.E., 29, 493 (1941).

${ }^{16}$ L. Infeld, Univ. of Toronto, Radiation Theory, Report No. 2 (1942).

${ }^{17}$ J. L. Synge, Univ. of Toronto, Radiation Theory, Report No. 4 (1942). 
In so far as the formal theoretical analysis is concerned, there is no restriction regarding the gap length $2 \delta$. In practice, one-dimensional circuit impedances are useful primarily for terminals that are close together compared with the wavelength. Hence, a reasonable restriction on the gap is

$$
\beta_{0} \delta \ll 1 .
$$

Subject to (24) it is possible to expand the current (22) in a Maclaurin series in powers of the small quantity $\beta_{0} \delta$. For sufficiently small values only the first two terms are required, viz.,

$$
I_{z}\left(\beta_{0} \delta\right) \doteq\left[I_{z}\left(\beta_{0} \delta\right)\right]_{\delta=0}+\beta_{0} \delta\left[\frac{\partial I_{z}\left(\beta_{0} \delta\right)}{\partial \beta_{0} \delta}\right]_{\delta=0} .
$$

The current at the driving-point is at $z=\delta$. Hence, setting $z=\delta$ in (25),

$$
I_{\delta}\left(\beta_{0} \delta\right)=I_{0}(0)+\beta_{0} \delta\left[\frac{\partial I_{\delta}\left(\beta_{0} \delta\right)}{\partial \beta_{0}}\right]_{\delta=0} .
$$

In (26), $I_{0}(0)$ is the driving point current calculated by King and Middleton and given in Ref. 1 by equation (24). Thus,

$$
I_{\delta}\left(\beta_{0} \delta\right)=I_{0}(0)[1-\epsilon],
$$

where

$$
\epsilon \equiv \epsilon^{\prime \prime}-j \epsilon^{\prime} \equiv-\frac{\beta_{0} \delta}{I_{0}(0)}\left[\frac{\partial I_{\delta}\left(\beta_{0} \delta\right)}{\partial \beta_{0} \delta}\right]_{\delta=0} .
$$

In expanded form, ${ }^{18}$

$$
\begin{aligned}
\epsilon= & \beta_{0} \delta\left\{\frac{\sin \beta_{0} h+G_{1}(h) / \psi+G_{2}(h) / \psi^{2}+\cdots}{\cos \beta_{0} h+F_{1}(h) / \psi+F_{2}(h) / \psi^{2}+\cdots}\right. \\
& +\frac{2}{\psi} \cdot \frac{e^{-i \beta_{0} h}}{\beta_{0} h} \cdot \frac{\left(1-\cos \beta_{0} h\right)+\left[F_{1}(0)-F_{1}(h)\right] / \psi+\cdots}{\cos \beta_{0} h+F_{1}(h) / \psi+F_{2}(h) / \psi^{2}+\cdots} \\
& \left.+\frac{2}{\psi} \cdot \frac{e^{-j \beta_{0} h}}{\beta_{0} h} \cdot \frac{\sin \beta_{0} h+G_{1}(h) / \psi+\cdots}{\sin \beta_{0} h+M_{1}(0) / \psi+M_{2}(0) / \psi^{2}+\cdots}\right\} .
\end{aligned}
$$

The impedance (23) may be obtained from (25) by noting that

$$
Z_{\delta} / Z_{0}=I_{0}(0) / I_{\delta}(\delta)=1 /(1-\epsilon),
$$

so that,

$$
\begin{aligned}
& R_{\delta}=\frac{R_{0}\left(1-\epsilon^{\prime \prime}\right)+X_{0 \epsilon^{\prime}}}{\left(1-\epsilon^{\prime \prime}\right)^{2}+\left(\epsilon^{\prime}\right)^{2}}, \\
& X_{\delta}=\frac{X_{0}\left(1-\epsilon^{\prime \prime}\right)-R_{0} \epsilon^{\prime}}{\left(1-\epsilon^{\prime \prime}\right)^{2}+\left(\epsilon^{\prime}\right)^{2}} .
\end{aligned}
$$

${ }^{18}$ The differentiation is straightforward; use is made of the relation $F_{1}(z) \sin \beta_{0} h-G_{1}(z) \cos \beta_{0} h=0$ with $F_{1}(z)$ and $G_{1}(z)$ as in Ref. 1. 


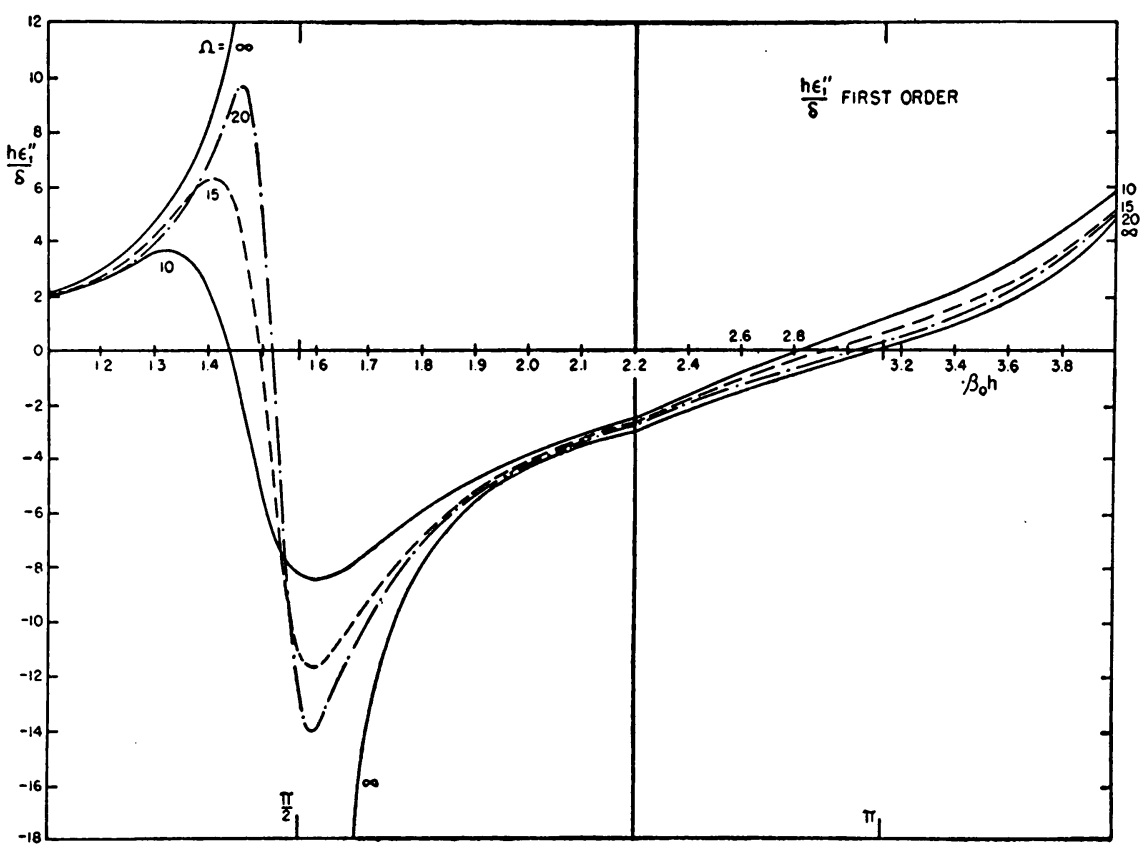

FIG. 3. First order correction factor $\epsilon_{1}^{\prime \prime}$.

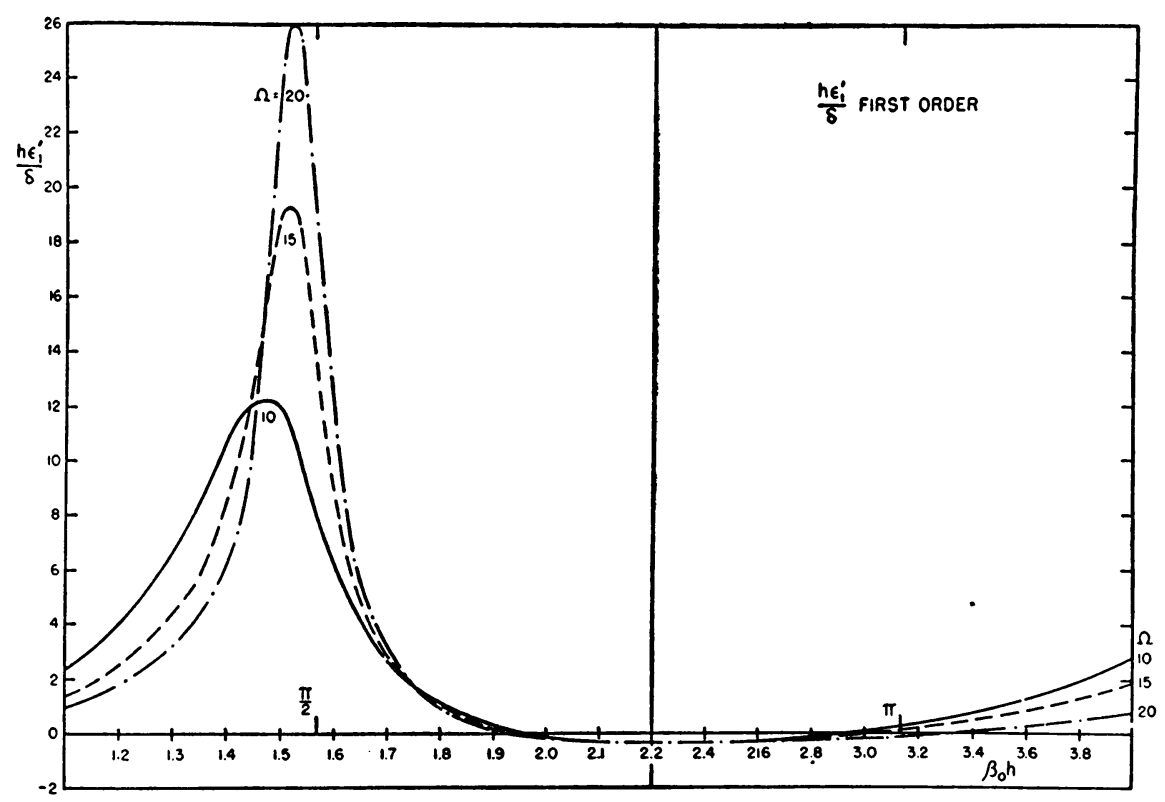

Fig. 4. First order correction factor $\epsilon_{1}^{\prime}$. 


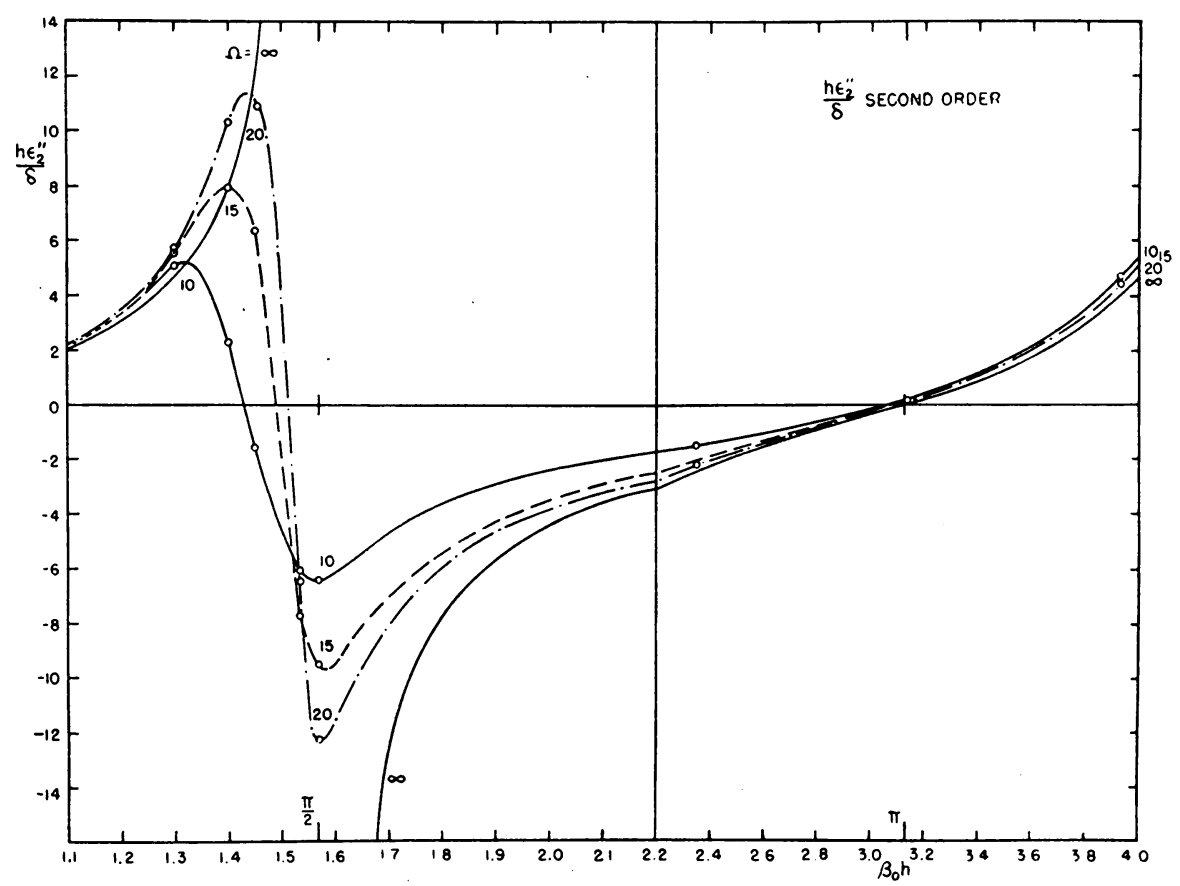

FIG. 5. Second order correction factor $\epsilon_{2}^{\prime \prime}$. Calculated points are shown; extrapolation below $\beta_{0} h=1.3$ is estimated using first order curves.

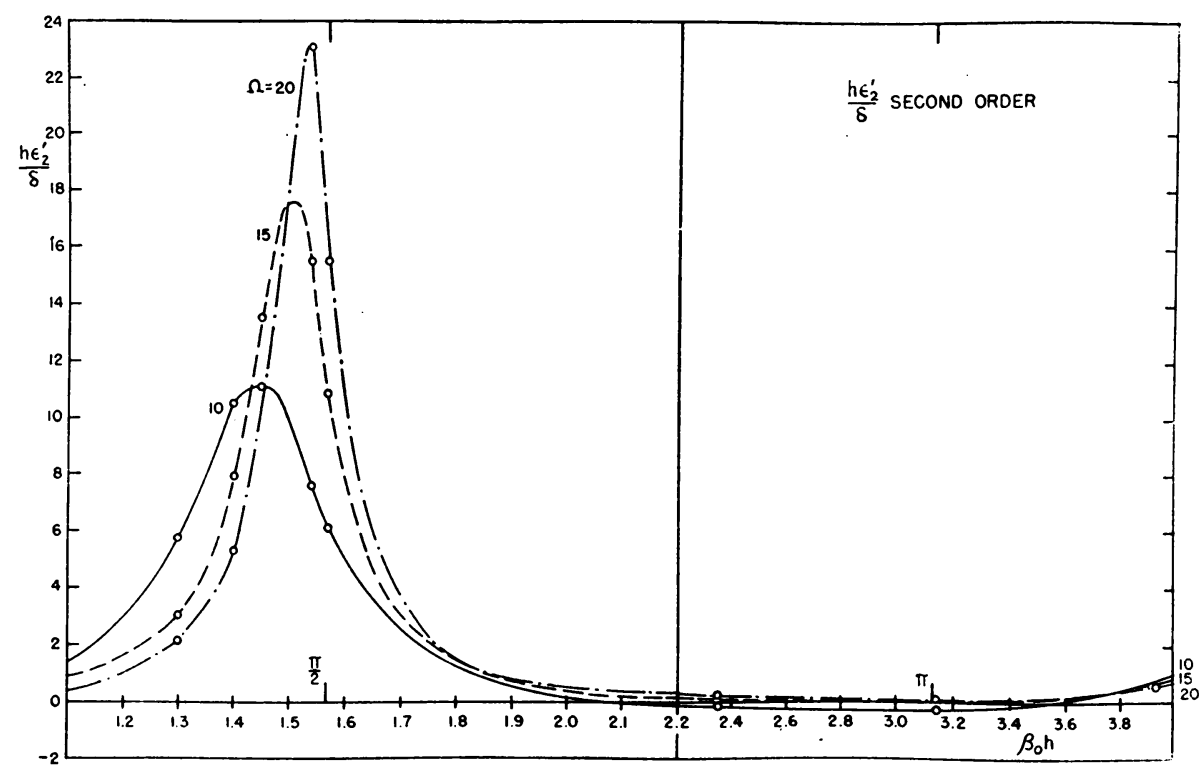

FIG. 6. Second order correction factor $\epsilon_{2}^{\prime}$. Calculated points are shown; extrapolation below $\beta_{0} h$ is estimated using first order curves. 


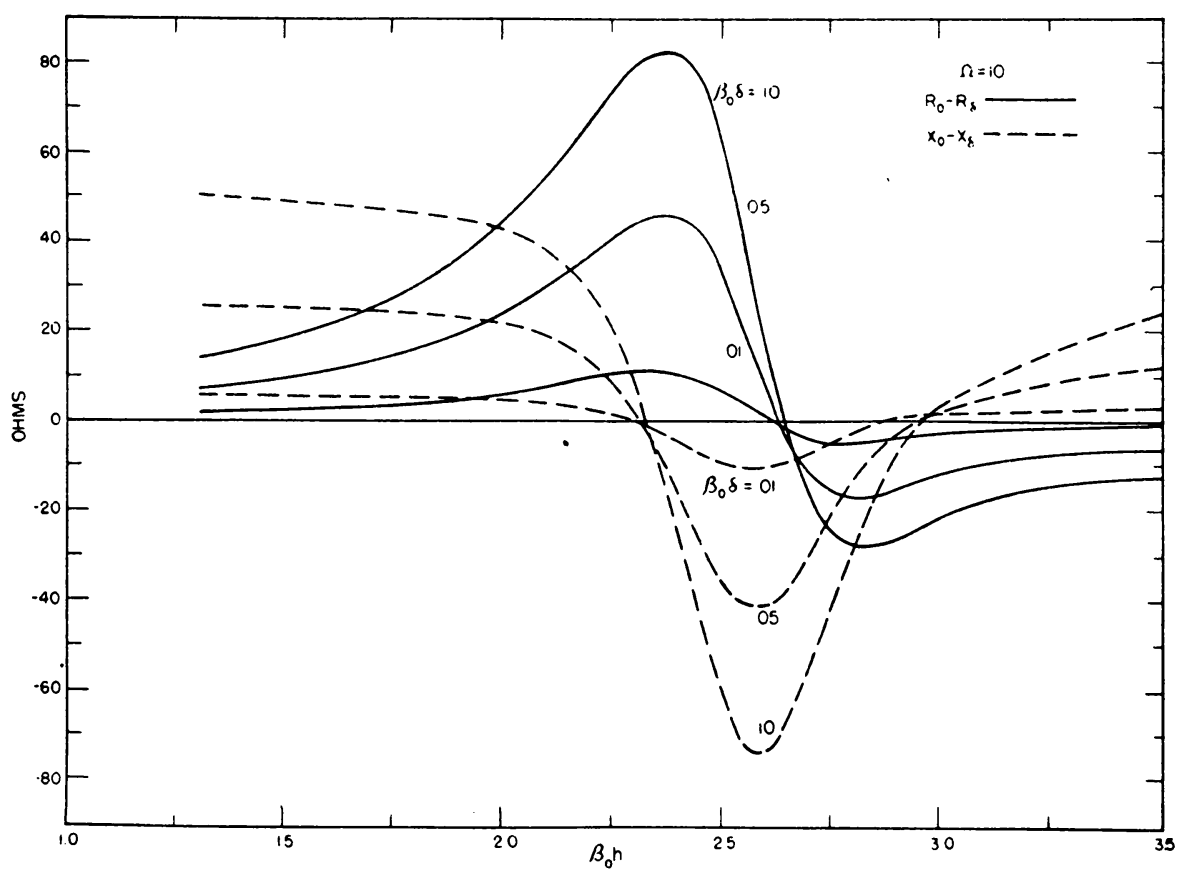

FIG. 7. Second order $R_{0}-R_{\delta}, X_{0}-X_{\delta}$ for several values of $\beta_{0} \delta$ with $\Omega=2 \ln (2 h / a)=10$.

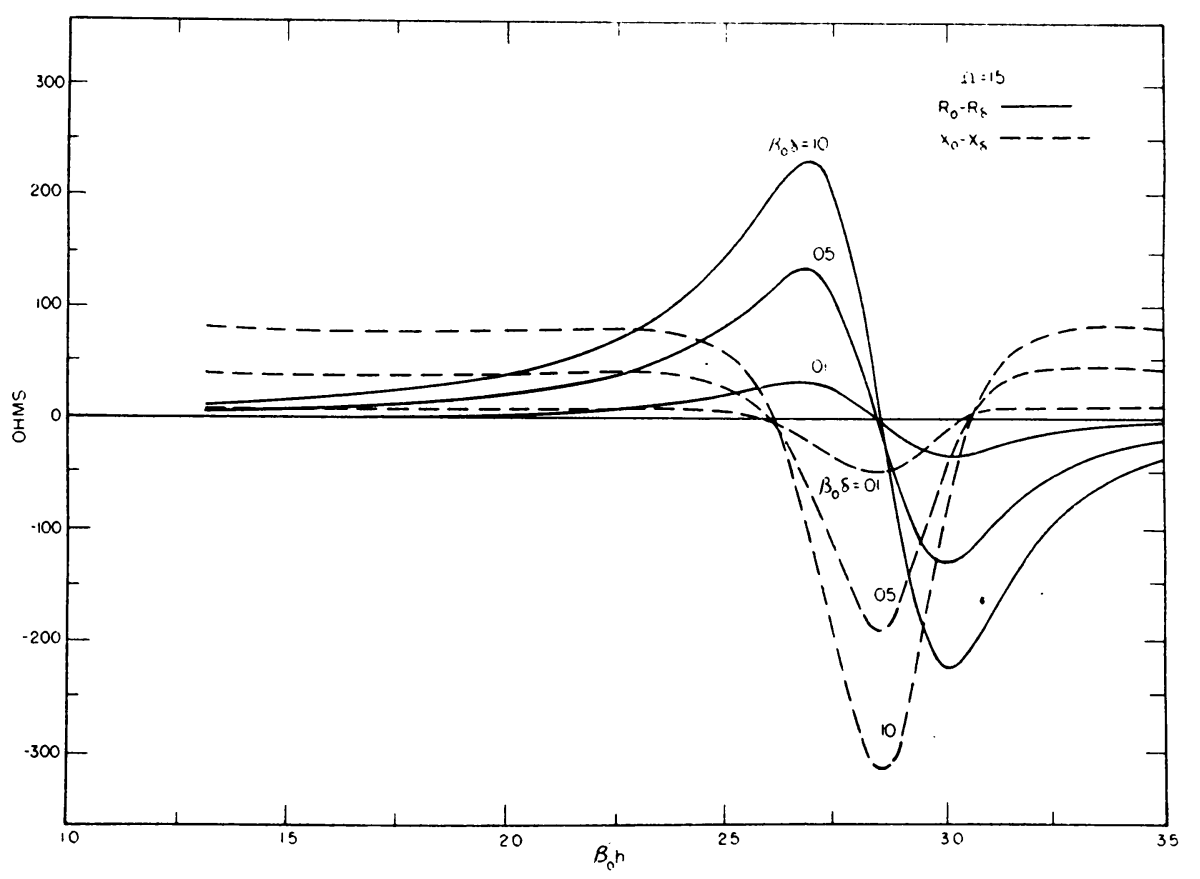

FIG. 8. Same as Fig. 7 with $\Omega=15$. 


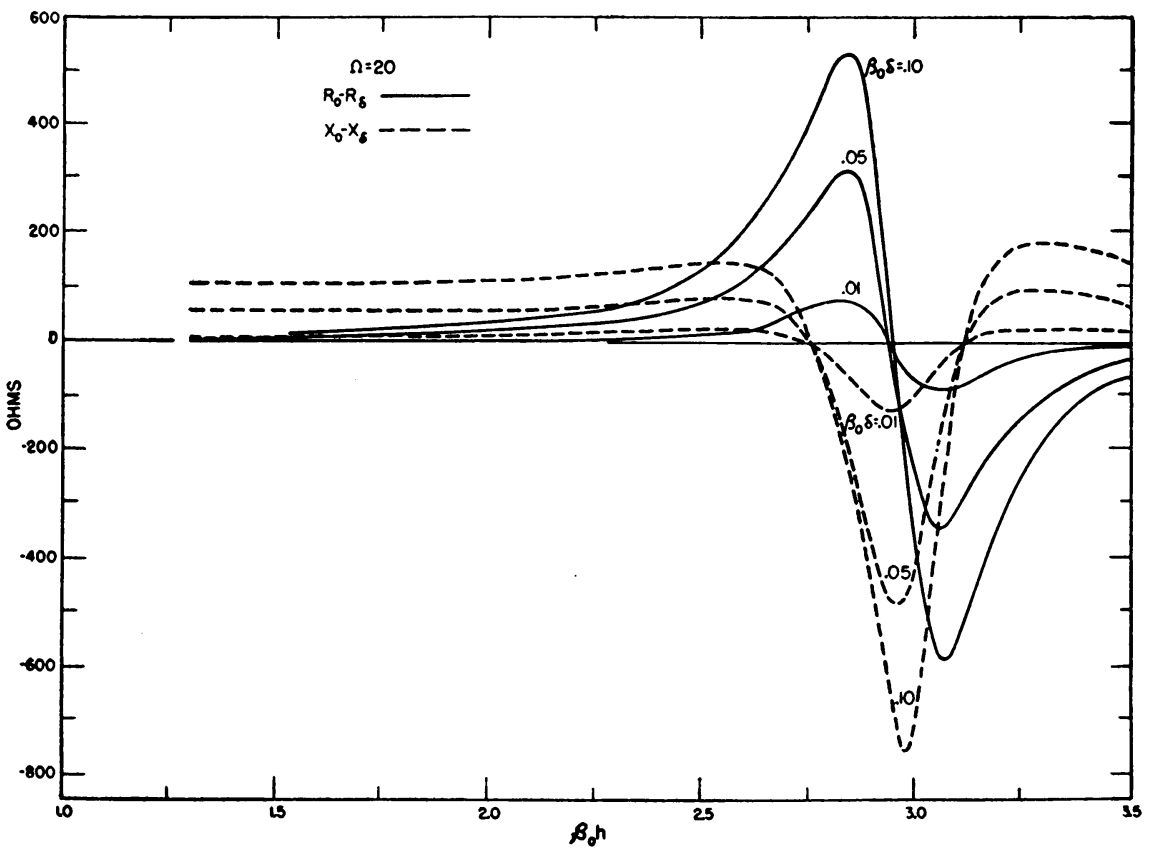

FIG. 9. Same as Fig. 7 with $\Omega=20$.

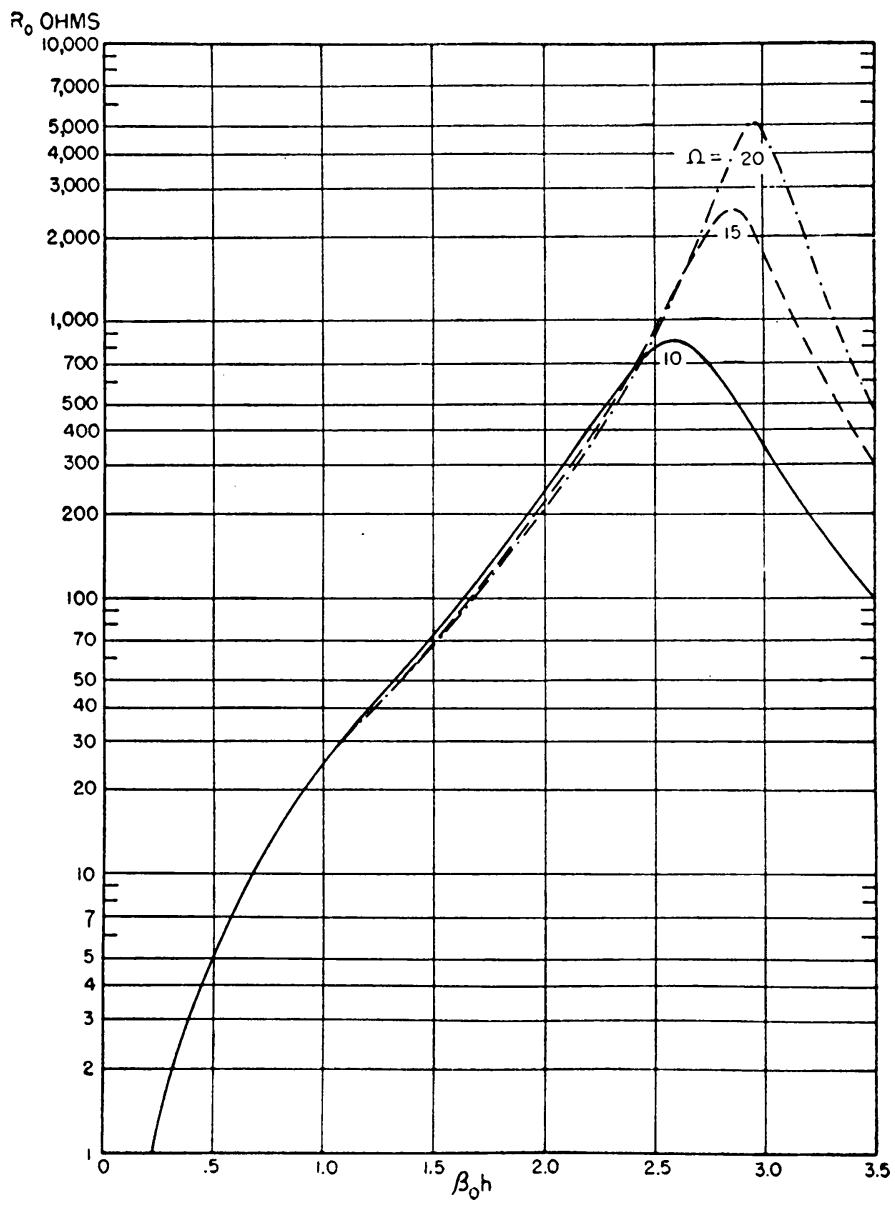

Fig. 10. Second order $R_{0}$ as a function of $\beta_{0} h$. 


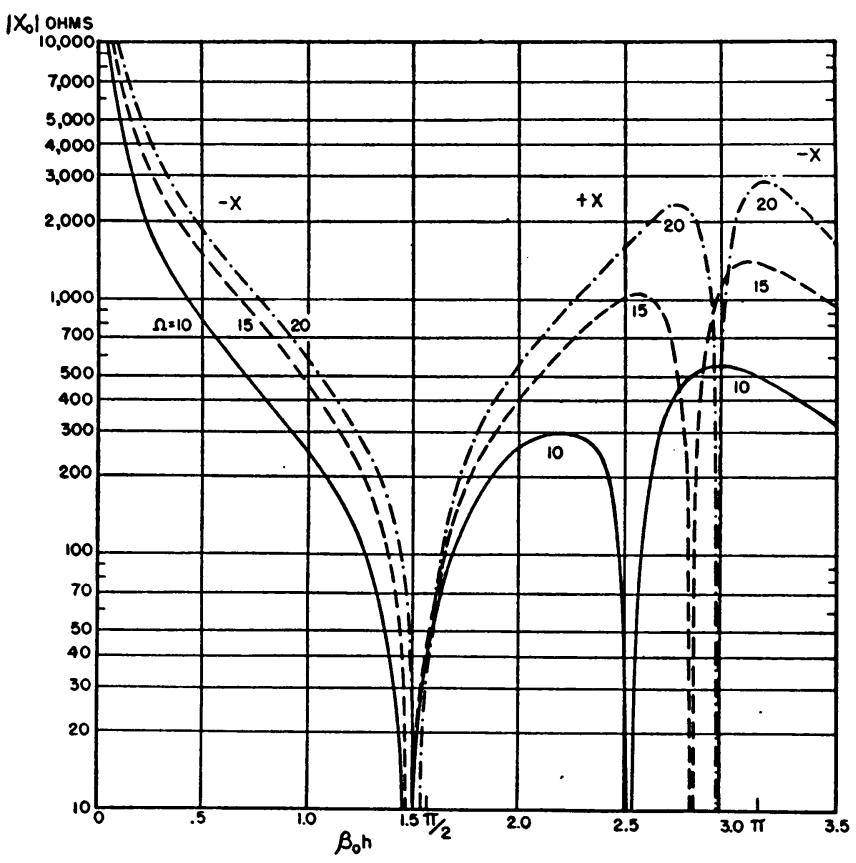

Fig. 11. Second order $X_{0}$ as a function of $\beta_{0} h$. (Small errors in the curves of Figs. 17-19 in Ref. 1 have been corrected in these curves.)

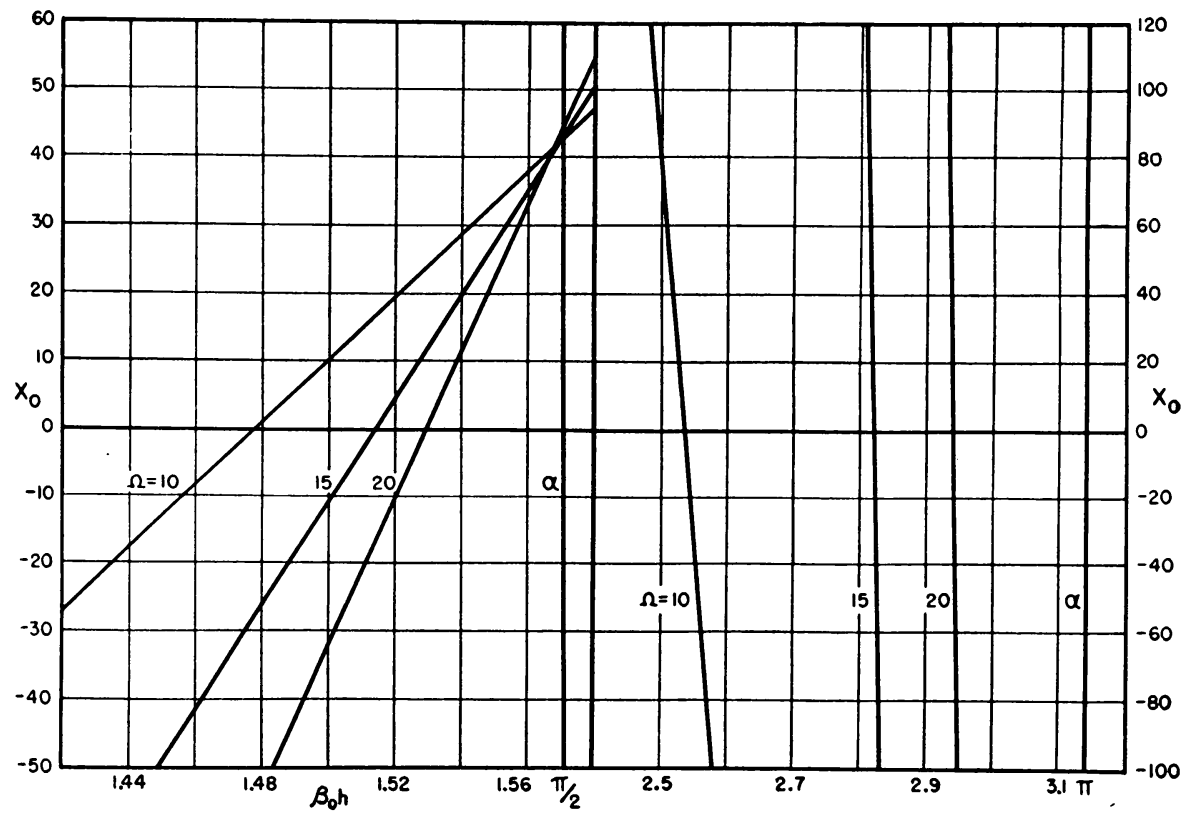

Fig. 12. Second order $X_{0}$ near resonance and anti-resonance. 

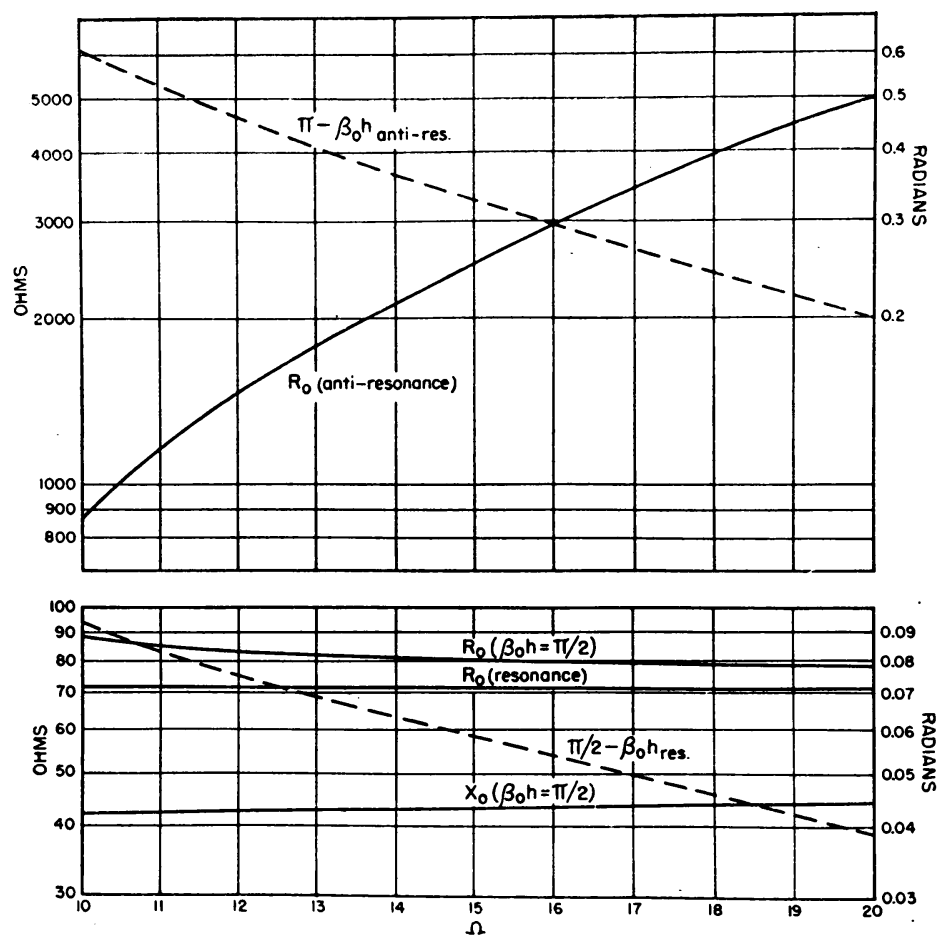

FIG. 13. Second order resonant and anti-resonant values of $R_{0} ; R_{0}$ and $X_{0}$ at $\beta_{0} h=\pi / 2 ; \pi / 2-\beta_{0} h_{\text {res. }}$. and $\pi-\beta_{0} h_{\text {anti-res. }}$ as functions of $\Omega$.

The first and second order correction factors in the form $\delta \epsilon / h$ are shown in Figs. 3-6 as functions of $\beta_{0} h$ for three values of $\Omega=2 \ln (2 h / a)$. It is seen that the correction is significant primarily near $\beta_{0} h=\pi / 2,3 \pi / 2$, etc. Curves showing second order values of $R_{0}-R_{\delta}$ and $X_{0}-X_{\delta}$ are found in Figs. 7-9 for several values of $\beta_{0} \delta$ and with $\Omega=10,15,20$.

5. Conclusion. The King-Middleton theory for the cylindrical antenna has been generalized to show the effect of a finite gap on the current and the impedance. The analysis shows that for small gaps the impedance is not very sensitive to gap length so that impedances calculated for zero gap are good approximations for a large class of antennas for which $\beta_{0} \delta<0.01$. If $\beta_{0} \delta \geqq 0.01$ the correction curves of Figs. 7-9 may be used in conjunction with the curves of Figs. $10-13$ for $R_{0}$ and $X_{0}$. It is interesting to note that for $\beta_{0} h$ near $\pi / 2$ the impedance $Z_{\delta}$ corrected for the gap differs negligibly from the uncorrected value $Z_{0}$ if the actual conductor half-length $h-\delta$ is used instead of $h$. This is not true for $\beta_{0} h$ near $\pi$. 